\title{
Revealing intrinsic and extrinsic piezoelectric contributions in phase coexistence system of $\mathrm{PbTiO}_{3}-\mathrm{BiScO}_{3}$
}

\author{
Shengdong Sun ${ }^{1,2}$, Hui Liu ${ }^{3 *}$, Yang Ren ${ }^{5}$, Manuel Hinterstein ${ }^{4 *}$ and Jun Chen ${ }^{1,2,3 *}$
}

\begin{abstract}
PbTiO}_{3}-\mathrm{BiScO}_{3}$ ceramics possess high piezoelectricity and high Curie temperature simultaneously, presenting a very promising candidate for high-temperature actuators and transducers. Figuring out the underlying piezoelectric mechanisms is of great importance for its application. Herein, we reveal the quantified intrinsic and extrinsic contributions to the high electrostrain of the morphotropic composition with phase coexistence by employing the advanced in situ electrical biasing high-energy synchrotron $\mathrm{X}$-ray diffraction combined with the STRAP (strain, texture, and Rietveld analysis for piezoceramics from diffraction) methodology. An electric field-induced phase transformation is observed between the coexisting tetragonal and rhombohedral phases. It is found that the tetragonal phase contributes most of the domain switching strain, while the predominant lattice strain is from the rhombohedral phase. With phase fraction changes, the quantitative results demonstrate that the fieldinduced electrostrain is mainly contributed by the intrinsic lattice strain. In contrast, the high irreversible domain switching strain dominates the large remanent strain. It implies that transforming the large remanent strain into reversible strain would be a strategic direction to improve the piezoelectric response. The present results provide a further understanding of the high piezoelectricity and could help to advance the application of $\mathrm{PbTiO}_{3}-\mathrm{BiScO}_{3}$.
\end{abstract}

Keywords: $\mathrm{PbTiO}_{3}-\mathrm{BiScO}_{3}$, piezoelectricity, in situ, synchrotron diffraction, electrostrain

\section{INTRODUCTION}

High-temperature piezoelectric ceramics demonstrate promising applications in actuators, ultrasonic transducers, energy harvesters, and fuel injection valves [1-3]. Although the traditional lead zirconate titanate (PZT)-based materials still dominate the market of piezo-devices, a secure working temperature, usually much lower than $200^{\circ} \mathrm{C}$, limits its development in the hightemperature piezoelectric field. The $\mathrm{PbTiO}_{3}-\mathrm{BiScO}_{3}$ (PT-BS) system exhibits a high piezoelectric coefficient $\left(d_{33}=450 \mathrm{pC} \mathrm{N}^{-1}\right)$ and high Curie temperature $\left(T_{\mathrm{c}} \sim 450^{\circ} \mathrm{C}\right)$ simultaneously at the morphotropic phase boundary (MPB) [4], and is therefore considered as the most potential high-temperature piezoelectric ceramic. Recently, a broad range of related piezoelectric devices have been designed [5], trying to push PT-BS into practical application. Therefore, it is crucial to elucidate the underlying piezoelectric mechanisms of PT-BS, which is the cornerstone of the material application.

The enhanced piezoelectricity is usually reached near the $\mathrm{MPB}$, where the phase coexistence allows more possibilities of domain variants. Benefiting from advanced technologies, models such as polarization rotation [6], nanodomains [7], and electric field-induced phase transformation [8] have been proposed to rationalize the enhanced piezoelectricity. As for the important PT-BS, numerous studies have been conducted to understand its high piezoelectric performance. It seems that phase transformation is the most common contribution according to both ex situ and in situ results [8-11]. The phase coexistence of tetragonal and rhombohedral or monoclinic phases has been intensively studied using both neutron powder diffraction and transmission electron microscopy [12-15]. With the application of in situ techniques, the electric field-induced transformation between the coexisting phases can be directly investigated. It is believed that the significantly increased piezoresponse of PT-BS at the MPB is ascribed to the coupled domain wall motion, lattice strain, and phase transformation $[9,16]$. Despite these advances, the mechanism for its high piezoelectric performance is still not well understood, in particular, the detailed quantified intrinsic and extrinsic piezoelectric contributions of the individual phases in compositions close to the MPB. It is extremely challenging to quantitatively describe the behaviors of different components due to the rather complex structure near the MPB, such as phase coexistences and their interactions with each other, highly textured domains after poling, phase transformations, and intergranular interactions [17]. Recently, we have successfully developed a comprehensive method of STRAP (strain, texture, and Rietveld analysis for piezoceramics from diffraction), which is capable of extracting all the structural information for each coexisting phase at the MPB from in situ diffraction data, so that the individual contributions from different phases to the overall piezoresponse under in situ electric field can be weighted $[18,19]$. By using the recent methodology of STRAP, the strain mechanism of technically applied PIC151 [18] and $\left(\mathrm{Bi}_{1 / 2} \mathrm{Na}_{1 / 2}\right) \mathrm{TiO}_{3}-\mathrm{BaTiO}_{3}$ [20] ceramics have been quantitatively revealed from the perspective of lattice strain

\footnotetext{
${ }^{1}$ Beijing Advanced Innovation Center for Materials Genome Engineering, University of Science and Technology Beijing, Beijing 100083, China

${ }^{2}$ Department of Physical Chemistry, University of Science and Technology Beijing, Beijing 100083, China

${ }^{3}$ School of Mathematics and Physics, University of Science and Technology Beijing, Beijing 100083, China

${ }^{4}$ Institute of Applied Materials, Ceramic Materials and Technologies, Karlsruhe Institute of Technology 76131, Karlsruhe, Germany

${ }^{5}$ X-Ray Science Division, Advanced Photon Source, Argonne National Laboratory, Argonne, Illinois 60439, US

*Corresponding authors (emails: huiliu@ustb.edu.cn (Liu H); manuel.hinterstein@kit.edu (Hinterstein M); junchen@ustb.eud.cn (Chen J))
} 
$[21,22]$, domain switching [21,22], and phase transformation $[23,24]$.

In this work, we apply in situ synchrotron X-ray diffraction (SXRD) combined with the STRAP method to a morphotropic composition of the high-temperature PT-BS system. The structural parameters of individual phases were obtained and the corresponding contribution from the intrinsic lattice strain, extrinsic domain switching, and phase transformation was calculated quantitatively. The overall macroscopic strain was reconstructed flawlessly, which is consistent well with macroscopic measurements. We found that the intrinsic lattice strain of coexisting tetragonal and rhombohedral phases contributes to more than $60 \%$ of its field-induced electrostrain. The large macroscopic remanent strain is mainly ascribed to the high irreversible domain switching strain. Our results give insight into the strain mechanisms of high-temperature PT-BS ceramics and lay foundation for its application.

\section{EXPERIMENTAL SECTION}

\section{Material preparation and characterizations}

The ceramics of $0.64 \mathrm{PbTiO}_{3}-36 \mathrm{BiScO}_{3}$ (PT-36BS) were prepared using a conventional solid-state reaction method [4,5]. Highpurity $\mathrm{PbO}$ (99.9\%), $\mathrm{TiO}_{2}$ (99.8\%), $\mathrm{Bi}_{2} \mathrm{O}_{3}$ (99.99\%), and $\mathrm{Sc}_{2} \mathrm{O}_{3}$ (99.99\%) (Aladdin) powders were firstly baked at $200^{\circ} \mathrm{C}$ for $24 \mathrm{~h}$ to control the moisture. The raw materials were weighted stoichiometrically and ball-milled for $12 \mathrm{~h}$. The mixture was dried and calcined at $800^{\circ} \mathrm{C}$ for $5 \mathrm{~h}$ and then the calcined powder was milled for additional $12 \mathrm{~h}$. Subsequently, the powder was pressed into disks at the pressure of about $200 \mathrm{MPa}$. The disks were covered with powder of the same composition in a sealed alumina crucible and sintered at $1150^{\circ} \mathrm{C}$ for $2 \mathrm{~h}$. The well-sintered ceramics with a diameter of $8 \mathrm{~mm}$ were polished to a thickness of about $0.70 \mathrm{~mm}$, and then gold electrodes of approximately $60 \mathrm{~nm}$ were sputtered on both round surfaces. The direct macroscopic strain $(S-E)$ and the hysteresis loops were measured by a ferroelectric analyzer (TF1000, aixACCT, Germany). For the dielectric and piezoelectric measurements, the ceramics were first poled at $80^{\circ} \mathrm{C}$ for $30 \mathrm{~min}$ in silicone oil with an electric field of $5 \mathrm{kV} \mathrm{mm}^{-1}$. The poled ceramics were aged for $24 \mathrm{~h}$, and the small-signal $d_{33}$ was determined by a quasi-static $d_{33}$ meter (ZJ-3, China Academy of Acoustics). Temperature dependence of dielectric properties was measured by a multi-frequency inductance capacitance resistance analyzer (E4990, Agilent, Santa Clara, CA) at different frequencies. For the grain size measurement, the ceramic was polished and then thermal-etched at $800^{\circ} \mathrm{C}$ for $5 \mathrm{~min}$. Subsequently, the microstructure of the ceramic surface was characterized by scanning electron microscopy (SEM, S4800, HITACHI).

For the in situ experiment, the ceramics were cut into rectangular bars of $0.7 \mathrm{~mm} \times 1.2 \mathrm{~mm} \times 7.0 \mathrm{~mm}$ with electrodes on the long sides. The in situ electric field high-energy SXRD experiments were carried out at the 11-ID-C beamline at the advanced photon source (APS) of Argonne National Laboratory. The beam energy of $111.3 \mathrm{keV}(\lambda=0.11165 \AA)$ was used, taking the absorption of heavy elements of $\mathrm{Pb}$ and $\mathrm{Bi}$ into consideration. The sample was poled previously with several cycles. The electric field was applied at a step of $0.5 \mathrm{kV} \mathrm{mm}^{-1}$ from 5 to $0 \mathrm{kV} \mathrm{mm}^{-1}$. It should be noted that the zero-field state in this work refers to the remanent state. The transmission geometry was adopted and a two-dimensional (2D) detector was used to collect the diffraction data. The detailed information of the setup can be found elsewhere [25].

\section{Analysis}

The $2 \mathrm{D}$ diffraction data was integrated into diffraction patterns using the fit $2 \mathrm{D}$ software at an interval of $10^{\circ}$, i.e., patterns with different angles of the electric field to the incident beam can be easily obtained from a single 2D data set [26]. The STRAP analysis was carried out using the MAUD (materials analysis using diffraction) software. Details can be found elsewhere $[18,19]$. Advantages are that the full pattern Rietveld refinement and texture analysis for multiphase can be executed simultaneously. All data sets with various electric fields and orientations contribute to the final result. In our case, a phase structure model with $P 4 \mathrm{~mm}$ and $R 3 \mathrm{~m}$ coexistence was adopted according to the phase diagram of PT-BS [12].

As mentioned before, by deconvoluting all contributions from each phase, the fundamental strain mechanisms can be revealed. Therefore, appropriate parameters must be selected to quantitatively describe the lattice strain, domain switching, and phase transformation. Different models are available to fit the strain and texture effects in MAUD. Here, the lattice strain was fitted with the WSODF (texture-weighted strain orientation distribution function) model, and the E-WIMV (extended-WilliamsImhof-Matthies-Vinel) algorithm was used for the domain switching effect. The phase transformation can be easily estimated using the scale factor according to the Rietveld refinement. Given the fact of phase coexistence, both lattice strain and domain switching strain should be weighted with the phase fraction of tetragonal and rhombohedral phases. In this way, the lattice strain $\left(S_{\mathrm{L}}\right)$ and strain from domain switching $\left(S_{\mathrm{D}}\right)$ can be calculated as

$S_{\mathrm{L}}=\xi_{\mathrm{T}} \times S_{(\mathrm{L}, \mathrm{T})}+\xi_{\mathrm{R}} \times S_{(\mathrm{L}, \mathrm{R})}$,

$S_{\mathrm{D}}=\xi_{\mathrm{T}} \times S_{(\mathrm{D}, \mathrm{T})}+\xi_{\mathrm{R}} \times S_{(\mathrm{D}, \mathrm{R})}$,

where $S$ is the strain, $\xi$ is the phase fraction, and the subscript L and $\mathrm{D}$ represent the lattice and the domain strains, $\mathrm{R}$ and $\mathrm{T}$ denote the rhombohedral and tetragonal phases. It should be noted that the irreversible strain in this work refers to the remanent strain, which is calculated at the remanent state. And the reversible strain means the field-induced strain, which is the difference between the corresponding values at the applied field state and the remanent state. $S_{\mathrm{D}}$ can be calculated using the following equation [27]:

$S_{\mathrm{D}}=\eta \frac{1}{2 \pi} \int_{\varphi=0}^{\pi / 2}\left[\Delta \mathrm{MRD}_{h k l}(\varphi) \cdot \cos ^{2} \varphi\right](\sin \varphi) \mathrm{d} \varphi$,

where $\triangle \mathrm{MRD}$ is the difference of the pole figure density $f(h k l)$ in MRD (multiples of random distribution) from its random state and $\varphi$ is the inclination from the electric field vector. $\eta$ denotes the lattice distortion, which can be calculated according to the formula:

$\eta_{\mathrm{T}}=\frac{c_{\mathrm{T}}}{a_{\mathrm{T}}}-1$

$\eta_{\mathrm{R}}=\frac{\sqrt{2} c_{\mathrm{H}}}{\sqrt{3} a_{\mathrm{H}}}-1$

where the $c_{\mathrm{T}}, a_{\mathrm{T}}$, and $c_{\mathrm{H}}, a_{\mathrm{H}}$ are the corresponding unit cell parameters of the tetragonal and rhombohedral (H-index) phases, respectively. 
The pole figure density $f(h k l)$ in MRD is an effective indicator of domain switching, and it can be calculated from the intensity change of the reflections related to the direction of the polarization vectors. The polarization vector is $\langle 00 l\rangle_{\mathrm{T}}$ in the tetragonal phase and $\langle h h h\rangle_{\mathrm{R}}$ in the rhombohedral phase. Thus, $200_{\mathrm{pc}}$ is a good indicator to investigate the domain switching in the tetragonal phase, and $111_{\mathrm{pc}}$ for the rhombohedral phase. The subscript $\mathrm{pc}$ indicates the pseudocubic indexing, while $\mathrm{T}$ and $\mathrm{R}$ indicate tetragonal and rhombohedral indexing, respectively. Usually, MRDs can be calculated using the ratios of the integrated intensities of the $h k l$ reflections at the remanent and the applied field states, respectively [28]. However, in this work, the MRDs were extracted from the pole figures calculated from the orientation distribution functions (ODFs) $[19,29]$. The complete ODFs are easily acquired according to the texture refinement with MAUD.

\section{RESULTS AND DISCUSSION}

The microstructure and electrical properties characterizations of PT-36BS ceramics are shown in Fig. 1. The morphology of the polished and thermal-etched ceramic surface shows the dense microstructure of the sintered ceramics. The average grain size is estimated to be $1.5 \mu \mathrm{m}$ (Fig. 1a, b). A high piezoelectric coefficient $d_{33}$ of $435 \mathrm{pC} \mathrm{N}^{-1}$ is determined for the prepared ceramics. Correspondingly, the $T_{\mathrm{c}}$ extracted from the temperature where the dielectric constant reaches the maximum is $440^{\circ} \mathrm{C}$ (Fig. 1c). The polarization-electric field $(P-E)$ loops as a function of the electric field indicate a coercive field of $2.3 \mathrm{kV} \mathrm{mm}^{-1}$ and a remanent polarization of $40 \mu \mathrm{C} \mathrm{cm}^{-2}$ (Fig. 1d). The observed piezoelectric properties agree well with those of previous reports
$[4,5]$.

The characteristic reflections of $110_{\mathrm{pc}}, 111_{\mathrm{pc}}$, and $200_{\mathrm{pc}}$ are plotted as a function of the electric field orientation (Fig. 2). An intuitive observation on the contour plots shows a phase coexistence nature, which can be easily seen from the multiple $200_{\mathrm{pc}}$ reflections. The relative weak intensity of the middle reflection between $200_{\mathrm{T}}$ and $002_{\mathrm{T}}$ indicates that the tetragonal phase is dominant in PT-36BS composition. It can also be corroborated by the Rietveld refinement results of powder XRD (see also Fig. S1). Therefore, the orientation-dependent shift of the $111_{p c}$ reflection results from the lattice strain and intergranular interaction. In the tetragonal phase, the $200_{\mathrm{pc}}$ reflection is a good indicator of domain texture. The intensity emerging around $2 \theta=$ $3.18^{\circ}$ should be the result of the phase coexistence, and it can be indexed as rhombohedral $200_{\mathrm{R}}$ reflection [12] (Fig. 2c).

With the applied electric field at $5 \mathrm{kV} \mathrm{mm}^{-1}$, the shift of the orientation-dependent reflection position increases (Fig. 2 and Fig. S2). It suggests that a strong electric field-induced lattice stain occurs. As for the domain switching, it is quite obvious that the intensity contrast between $002_{\mathrm{T}}$ and $200_{\mathrm{T}}$ is stronger in the applied field state than in the remanent state (Fig. $2 \mathrm{f}, \mathrm{l}$ ), indicating a field-induced reversible domain switching. While the orientation-dependent intensity variation of the $200_{\mathrm{pc}}$ reflection in the remanent state, as depicted in Fig. $2 \mathrm{f}$, is ascribed to irreversible domain switching upon poling. It is worth noting that the intensity of the $200_{\mathrm{R}}$ reflection increases distinctly with the applied electric field (Fig. 2f, l), which demonstrates a phase transformation from tetragonal to rhombohedral.

According to the above analysis, the Rietveld refinement and texture analysis with a structural model of $P 4 \mathrm{~mm}$ and $R 3 \mathrm{~m}$ using a

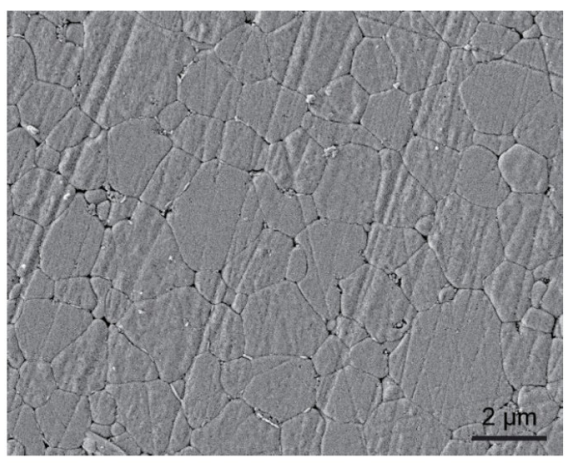

c

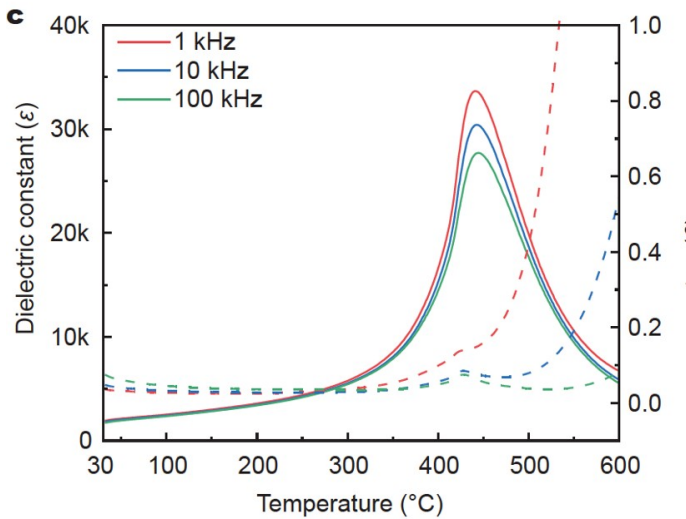

b
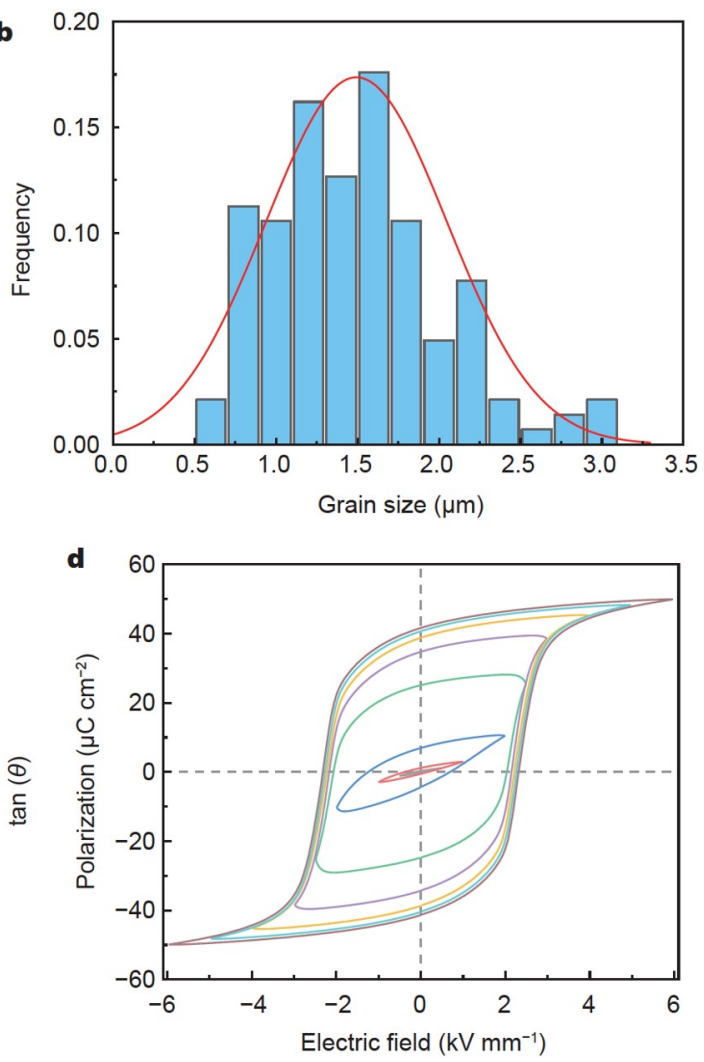

Figure 1 (a) The SEM image of the polished and thermal-etched ceramic surface. (b) The grain size distribution. (c) Temperature dependence of dielectric spectrum, and (d) P-E loops of PT-36BS. 

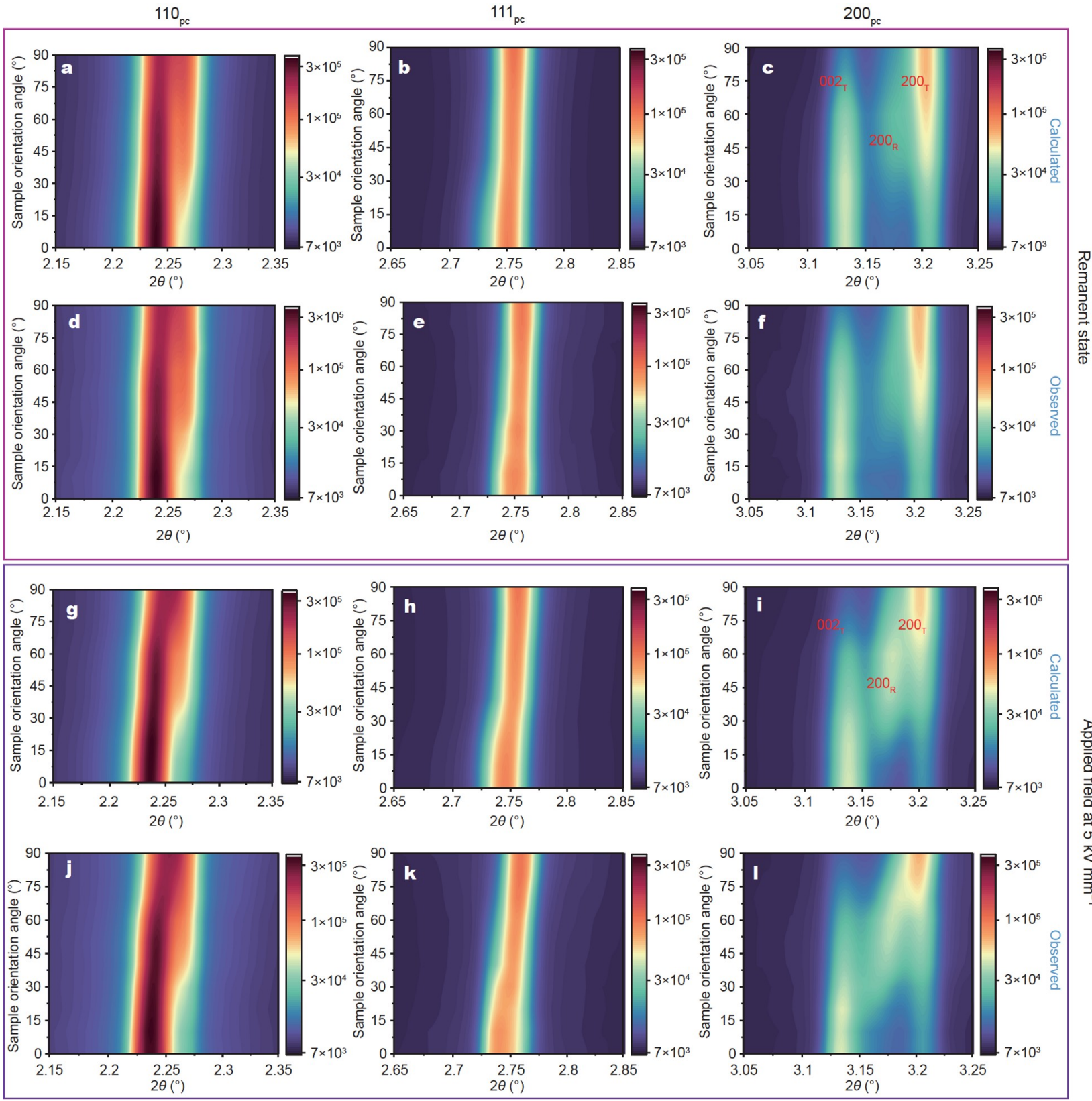

Figure 2 Plots of the $110_{\mathrm{pc}}, 111_{\mathrm{pc}}$ and $200_{\mathrm{pc}}$ reflections as a function of the electric field orientation. $(\mathrm{a}-\mathrm{c})$ Calculated diffraction patterns and (d-f) measured diffraction patterns for the remanent state at $0 \mathrm{kV} \mathrm{mm}^{-1}$. (g-i) Calculated diffraction patterns and $(\mathrm{j}-\mathrm{l})$ measured diffraction patterns for the applied field state at $5 \mathrm{kV} \mathrm{mm}^{-1}$.

the MAUD software worked flawlessly with the data. As depicted in Fig. 2, the bottom rows show the measured patterns and the top rows show the results of the refinement. A comparison between the top and the bottom rows verifies the accuracy of the structure model and the refinement (see also Figs S2 and S3).

Fig. 3 depicts the strain calculations from the refinement. It is quite obvious that the tetragonal phase is dominated by domain switching strain and the rhombohedral phase more by lattice strain. This is very similar to what was observed already in PZT [19]. The absolute overall strain is dominated by the domain switching strain. The increase in strain is not very pronounced anymore when the electric field exceeds $4 \mathrm{kV} \mathrm{mm}^{-1}$, which is consistent with the saturation state revealed by the macroscopic hysteresis loop (Fig. 1c).

A detailed comparison of the refinement parameters is listed in Table 1. The dominant tetragonal phase accounts for $77.9 \%$ at the remanent state and $66.3 \%$ at $5 \mathrm{kV} \mathrm{mm}^{-1}$. That is, the electric field induces $11.6 \%$ tetragonal phase to transform into rhombohedral (see also Fig. 4a). The tetragonal lattice strain is relatively small, with only $0.024 \%$ at the remanent state and $0.067 \%$ with the applied field, delivering a $0.043 \%$ field-induced lattice strain. In contrast, rhombohedral lattice strain is significantly higher, with $0.404 \%$ at the remanent state and $0.711 \%$ at $5 \mathrm{kV} \mathrm{mm}^{-1}$. As a result, the lattice strain contributed by the rhombohedral phase is more than seven times that of the tetragonal. When taking the phase fractions into consideration, the lattice strain accounts for $0.177 \%$ field-induced reversible strain. 
As for the domain switching strain, the tetragonal phase contributes $0.148 \%$ field-induced strain. This value is about seven times that of the rhombohedral $(0.021 \%)$, which is coincidently opposite to the situation of the lattice strain. As a result, domain switching strain accounts for $0.089 \%$ field-induced reversible strain.

Based on the calculations, the PT-36BS presents a strong remanent strain of $0.517 \%$ and a field-induced reversible strain of $0.266 \%$ (also see the Supplementary information). About $67 \%$ $(0.177 \%)$ field-induced reversible strain comes from the lattice strain. Although the rhombohedral phase is minor, its significant contribution to the field-induced strain cannot be ignored, especially for the lattice strain (Table 1). However, the reversibility of the rhombohedral domain switching is not significant. The domain switching strain is mainly from the tetragonal phase, which is consistent with the results reported in tetragonal La-doped PZT, where the domain switching plays a more important role in the overall strain than the lattice strain [30].

To elucidate the reasons for the different contributions from the tetragonal and rhombohedral phases, more detailed phase and structure information is necessary. As has been mentioned before, the tetragonal phase is dominant (Fig. 4a and Fig. S1). With increasing electric field, the tetragonal phase fraction decreases through phase transformation. It should be noted that the phase fraction here is an average result from all the orientations. The key factor causing the different roles of strain contribution between the tetragonal and rhombohedral phases is the significant difference in unit cell distortion. This can be seen in Fig. 4b, where the rhombohedral unit cell distortion stays constant at a level of about $0.6 \%$ during the application of the electric field, while the tetragonal unit cell distortion constantly decreases for increasing fields from $2.3 \%$ to $2.0 \%$. The lower unit cell distortion means that the strain from non- $180^{\circ}$ domain switching is quite limited. Instead, the small unit cell distortion should benefit the lattice strain, as has been revealed in Fig. 3b. As for the tetragonal phase, the larger unit cell distortion undoubtedly enhances its domain switching strain but inevitably resulting in a rather lower lattice strain (Fig. 3a). It is worth emphasizing that the existence of the rhombohedral phase could favor the considerable tetragonal domain switching and lattice strain with such a large unit cell distortion [31].

Additionally, the unit cell distortion of the rhombohedral phase stays constant with the applied electric field, while that of the tetragonal phase decreases from $2.3 \%$ to $2.0 \%$. The reason for this is that the refinement only yields a single set of unit cell parameters. These are an average over the whole sample volume for all orientations of domains. When the field is applied, the amount of tetragonal domains aligned along the field increases. However, the resulting $c$-axis length of the switched domains will be smaller than the initial one, because otherwise the switched grains would have to expand by the unit cell distortion. In the tetragonal case, this would be more than $2 \%$. The surrounding bulk and intergranular stresses prevent this and result in a decreased unit cell distortion. The rhombohedral unit cell distortion lies in the range of the macroscopic strain and therefore remains constant. A similar observation was recently reported for PZT [18].

Even though the rhombohedral unit cell distortion is significantly smaller than the tetragonal one, the rhombohedral domain switching strain is still relevant. The reason for this is
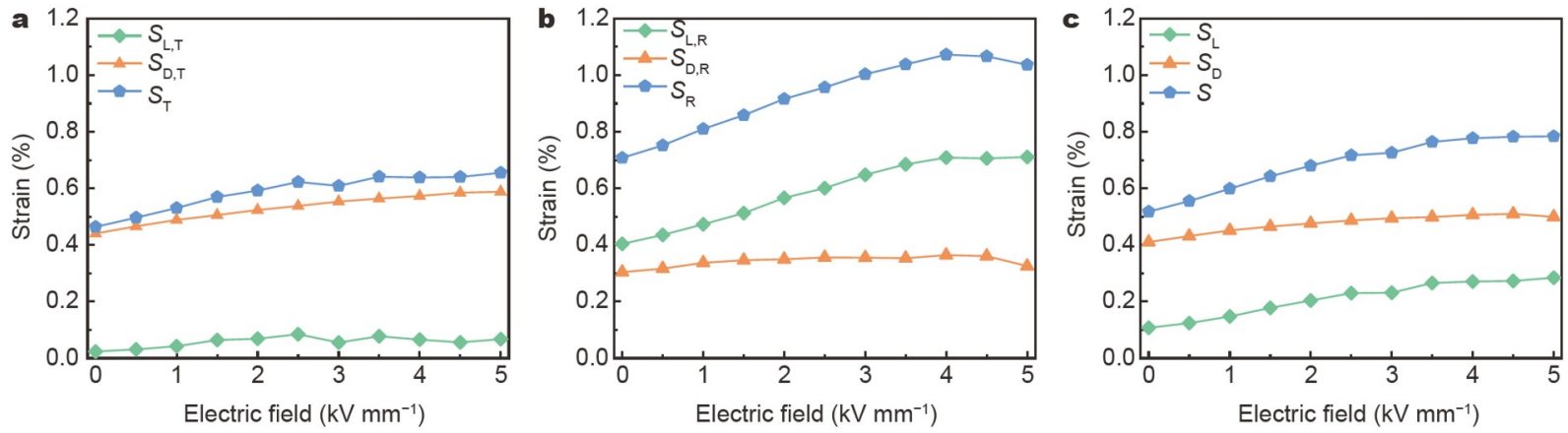

Figure 3 Strain calculations for the individual phases $(\mathrm{a}, \mathrm{b})$ and the combined values $(\mathrm{c})$. The subscript $\mathrm{L}$ denotes the lattice strain, D denotes the domain switching strain, and $\mathrm{T}$ and $\mathrm{R}$ denote the respective tetragonal and rhombohedral phases.
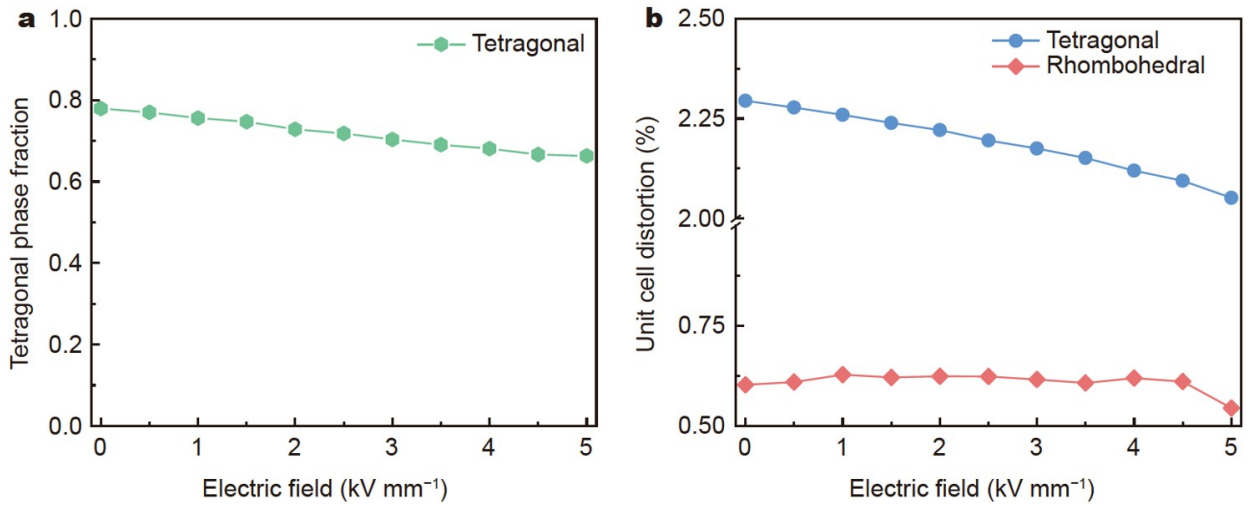

Figure 4 Tetragonal phase fractions (a) and unit cell distortions (b) of the tetragonal and the rhombohedral phase as a function of the electric field. 
the extraordinarily high degree of texture. The rhombohedral pole figure density $f(111)$ reaches values of almost 8 , which is the theoretical limit for a tetragonal and rhombohedral phase coexistence (Fig. 5b).

A closer look on $f(h k l)$ as a function of $\varphi$ shows that the pole figure densities of both tetragonal and rhombohedral phases reach their maximum at $\varphi=0^{\circ}$. That is, the strong texture state lies along the electric field direction, which is consistent with our intuition. With increasing $\varphi$, an almost non-texture state occurs at around $40^{\circ}-50^{\circ}$, where $f(h k l)$ are near 1 . This behavior is consistent with the theoretically calculated $f(h k l)$ [32] and those experimental results [25,33]. Also, that the MRD is near 1 in a specific angle range is the basis of those studies making Rietveld refinement using only the $45^{\circ}$ orientation $[25,34]$ without considering domain texture. It is clear that a strong texture state is maintained at the remanent state owing to the electric poling during the initial cycle of the electric field. The difference of $f(h k l)$ between the remanent and applied field states is the contribution of the field-induced reversible domain switching. The domain switching also results in the elongation of the materials along the vector of the electric field. The macroscopic strain caused by the domain switching can be calculated according to Equations (2)-(5). And the discussion has been provided in Fig. 2, Table 1, and the corresponding context.

Since the intensities vary strongly with orientation, it is impossible to deconvolute the phase fraction from the texturing directly. However, there is an indirect way of estimating the magnitude of the orientation-dependent phase transformation [19]. Fig. 6 shows a comparison of the pole figure densities $f(h k l)$ of the tetragonal $111_{\mathrm{T}}$ and the rhombohedral $200_{\mathrm{R}}$ reflections at the remanent and the applied field states. The $111_{\mathrm{T}}$ reflection in tetragonal symmetry and the $200_{\mathrm{R}}$ reflection in rhombohedral symmetry are not split. Since it is impossible to generate texturing in a single reflection of a former untextured material, the intensity is proportional to the phase fraction. However, due to the orientation-dependent phase transformation, the pole figure densities change. For the tetragonal phase, the change of pole figure densities is not very dramatic, but for the rhombohedral phase, it is (Fig. 6). This scenario suggests that the phase transformation here is mainly from the rhombohedral grains whose $\langle h h h\rangle_{\mathrm{pc}}$ polarization vectors deviate from the electric field direction. Whereas, $\langle 00 l\rangle_{\mathrm{pc}}$ axis of those rhombohedral domains could align along the electric field direction. In this case, a phase transformation to tetragonal symmetry favors an improved alignment of polarization vectors to the electric field. As for those tetragonal grains whose $\langle h h h\rangle_{\mathrm{pc}}$ axis align along the electric field direction, a change to rhombohedral symmetry improves the alignment of polarization similarly. A weaker response in orientation-dependent phase transformation could be ascribed to the large lattice distortion of the tetragonal phase (Fig. 4b), which makes the polarization rotation difficult. A strong remanent texture of the rhombohedral $f(200)_{\mathrm{R}}$ suggests that the orientation-dependent phase transformation is irreversible. With the applied electric field, a decrease of rhombohedral $f(200)_{\mathrm{R}}$ at the lower angle of $\varphi\left(\varphi<30^{\circ}\right)$ means the rhombohedral phase is transformed here, which coincides with the fieldinduced increase of tetragonal $f(200)_{\mathrm{T}}$ (Fig. 6a). A pronounced increase of the rhombohedral $f(200)_{\mathrm{R}}$ is observed near $\varphi=54^{\circ}$.

Table 1 The refinement results of PT-36BS at the remanent state at $0 \mathrm{kV} \mathrm{mm}^{-1}$ and the applied field state at $5 \mathrm{kV} \mathrm{mm}^{-1}$. Abbreviations are used to be consistent with the text.

\begin{tabular}{lcccc}
\hline & Parameters & Remanent state $\left(0 \mathrm{kV} \mathrm{mm}^{-1}\right)(\%)$ & Applied field $\left(5 \mathrm{kV} \mathrm{mm}^{-1}\right)(\%)$ & Difference $(\%)$ \\
\hline \multirow{2}{*}{ Phase fraction $(\eta)$} & $\eta_{\mathrm{T}}$ & 77.9 & 66.3 & -11.6 \\
& $\eta_{\mathrm{R}}$ & 22.1 & 33.7 & 11.6 \\
Lattice strain $\left(S_{\mathrm{L}}\right)$ & $S_{(\mathrm{L}, \mathrm{T})}$ & 0.024 & 0.067 & 0.043 \\
& $S_{(\mathrm{L}, \mathrm{R})}$ & 0.404 & 0.711 & 0.307 \\
& $S_{\mathrm{L}}$ & 0.107 & 0.284 & 0.177 \\
Domain strain $\left(S_{\mathrm{D}}\right)$ & $S_{(\mathrm{D}, \mathrm{T})}$ & 0.440 & 0.588 & 0.148 \\
& $S_{(\mathrm{D}, \mathrm{R})}$ & 0.304 & 0.325 & 0.021 \\
& $S_{\mathrm{D}}$ & 0.410 & 0.499 & 0.089 \\
\hline
\end{tabular}
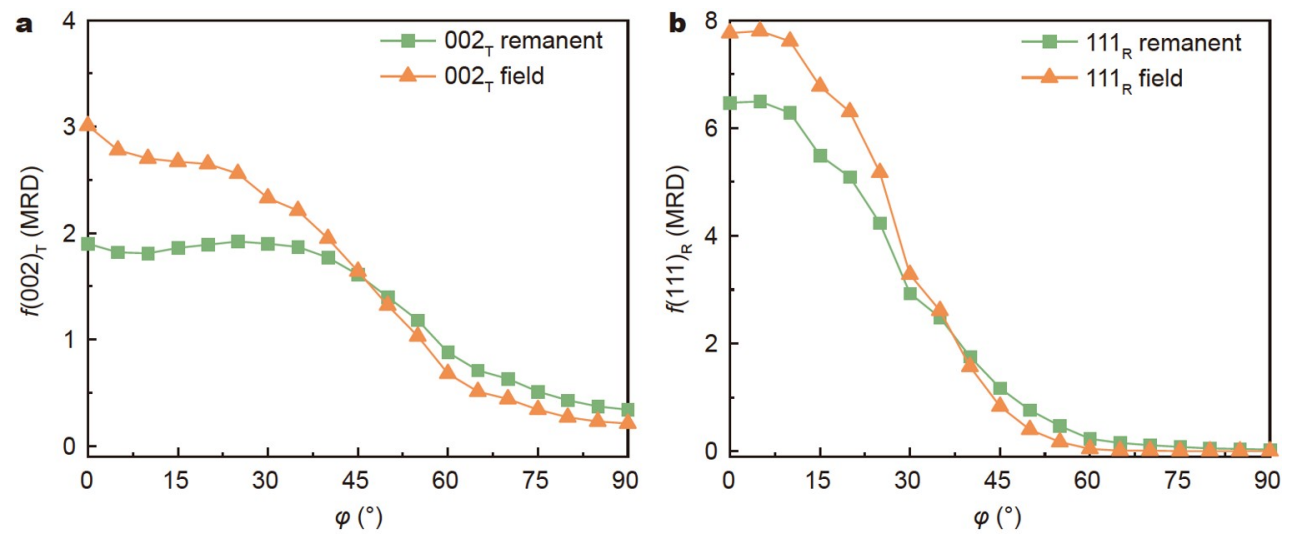

Figure $5 f(h k l)$ of the (a) $002_{\mathrm{T}}$ reflection for the tetragonal phase and (b) the $111_{\mathrm{R}}$ reflection for the rhombohedral phase at the remanent state $\left(0 \mathrm{kV}\right.$ mm $\left.{ }^{-1}\right)$ and the applied field state $\left(5 \mathrm{kV} \mathrm{mm}^{-1}\right) . \varphi$ is the angle with respect to the electric field vector. 
This suggests that the nature of orientation-dependent phase transformation is a polarization rotation between the $[001]_{\mathrm{T}}$ and $[111]_{R}$ polarization vectors $[19]$ because the angle between the polarization vectors of the tetragonal and rhombohedral phases also happens to be $54^{\circ}$.

Specifically, particular focus should be put on the strong remanent effect of the rhombohedral phase. The remanent state of $f(200)_{\mathrm{R}}$ demonstrates a strong texture state, indicating that the orientation-dependent phase transformation is not fully reversible (Fig. 6a). The high remanent value of $f(111)_{\mathrm{R}}$ leads to a smaller space of reversible domain switching, which has been verified in Fig. $3 \mathrm{~b}$ and Table 1 . As a result, only $0.021 \%$ fieldinduced domain switching strain is determined in the rhombohedral phase. Although a significantly high value of fieldinduced lattice strain is found in the rhombohedral phase, a considerable remanent lattice strain of $0.404 \%$ makes it potentially higher. All signs indicate that the field-induced strain would be even higher if it is possible to make the rhombohedral phase response reversible.

The macroscopic unipolar strain is depicted in Fig. 7. Considering the contribution of lattice strain, domain switching, and phase transformation, the overall remanent strain is calculated to be $0.517 \%$, and a field-induced strain of $0.266 \%$ is determined. Surprisingly, the corresponding macroscopic remanent strain is $0.513 \%$ at the initial poling cycle (Fig. $7 \mathrm{a}$ ), and the field-induced strain is $0.260 \%$ of the subsequent unipolar switching (Fig. $7 \mathrm{~b}$ ). Obviously, an excellent agreement has been reached between the refinement results and the macroscopic measurement. The results fully prove the feasibility of the model used in the STRAP method and the reliability of the data extracted from the refinement.

Based on the quantitative analysis results, the comparison of the calculated strain from different contributions and measured strain is shown in Fig. 8, where the remanent strain is deducted. It can be seen that the total calculated strain closely approximates the measured one. Besides, the lattice strain is about twice that of the domain switching strain. In other words, the fieldinduced strain mechanism of PT-36BS is dominant by intrinsic lattice other than extrinsic domain contribution. Note that in the present method, the contribution of phase transformation cannot be evaluated individually, since the phase transformation is coupled strongly with lattice strain and domain switching strain. As a matter of fact, the phase transformation contribution has been considered as an additional variable in Equations (1) and (2), because it is even harder to quantify the role of phase transformation if this is not the case. Phase transformation plays an important role in enhancing piezoelectricity as reported previously $[16,23]$.

Another thing worth noting is that high remanent strain (about $0.5 \%$ ) was observed and also confirmed by calculations in PT-36BS composition (Figs $3 \mathrm{c}$ and $7 \mathrm{a}$ ). Converting the remanent strain into a field-induced strain will significantly improve the converse piezoelectricity. Looking back at Fig. $3 c$, it is found that the remanent strain is mainly controlled by the extrinsic domain switching. For the tetragonal phase in ceramics, the large unit cell distortion constrains its non- $180^{\circ}$ domain switching. How-
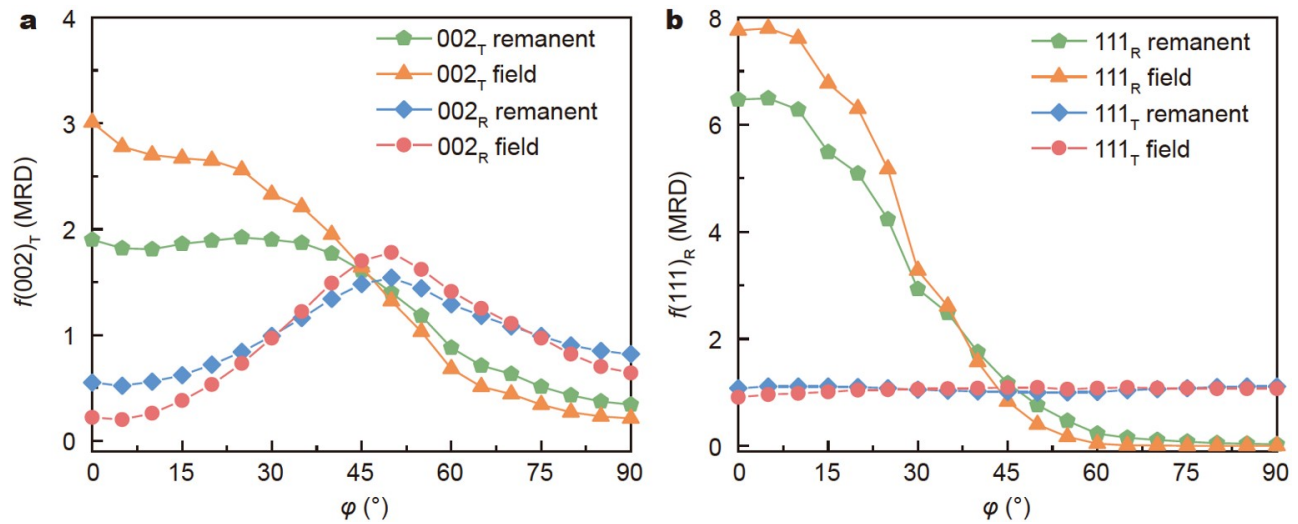

Figure 6 Plots of the (a) rhombohedral $f(200)_{\mathrm{R}}$ and (b) tetragonal $f(111)_{\mathrm{T}}$ compared with the $f(002)_{\mathrm{T}}$ and $f(111)_{\mathrm{R}}$ from Fig. 4 at the remanent state $\left(0 \mathrm{kV} \mathrm{mm}^{-1}\right)$ and the applied field state $\left(5 \mathrm{kV} \mathrm{mm}^{-1}\right)$.
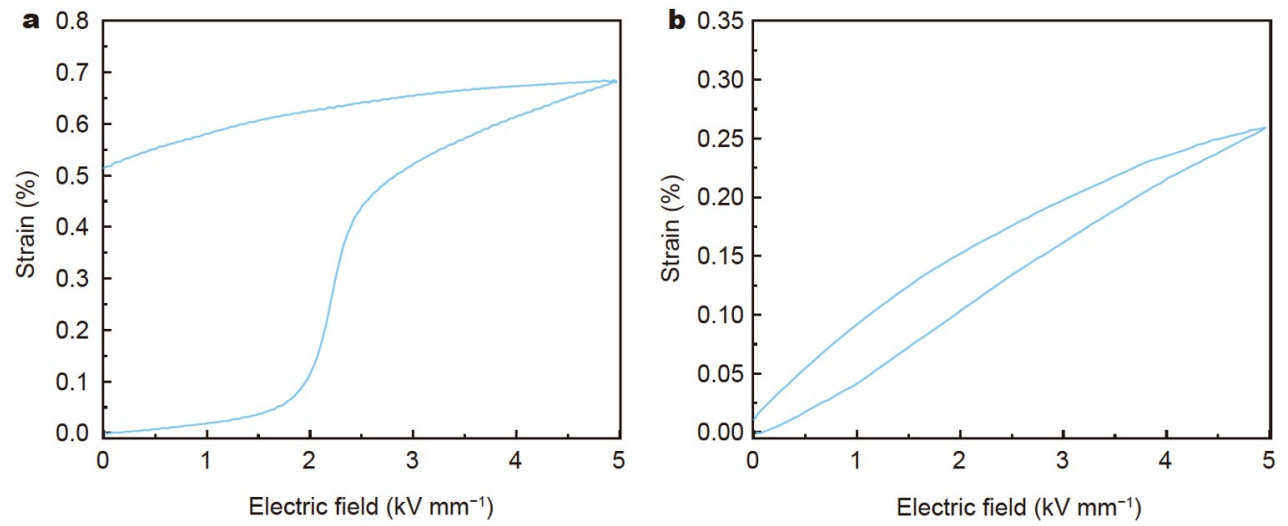

Figure 7 Macroscopic strains of (a) the initial poling cycle and (b) the unipolar switching. The frequency of the measurements is $30 \mathrm{mHz}$. 


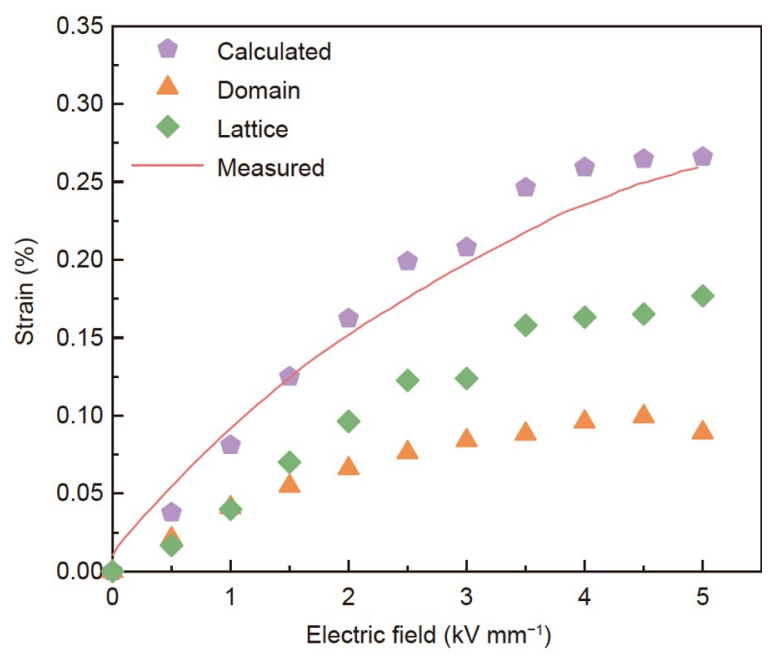

Figure 8 Comparison of the calculated strain and macroscopically measured strain.

ever, sacrificing the unit cell distortion to activate the domain switching will inevitably reduce the Curie temperature. Therefore, modifying the rhombohedral phase in ceramics might help. As discussed in Fig. 6, the strong remanent effect of the rhombohedral phase is not conducive to large reversible strain. Element doping could be an alternative strategy. Besides, the theoretical approach has demonstrated that reversible domain switching strain in a single tetragonal or rhombohedral polycrystalline ceramic is unlikely to happen [31]. Therefore, the existence of a secondary phase is likely to promote the ability of the domain switching strain mutually. From this point of view, controlling the relative phase fraction through precisely modifying the composition or improving the process should be effective. Additionally, it is also a feasible but challenging method to provide restoring force by constructing a core-shell structure [35].

\section{CONCLUSION}

The strain mechanism of high-temperature piezoceramic PT36BS has been systematically investigated by using in situ SXRD combined with the STRAP method. The electric field-induced phase structure and domain texture evolution were taken into consideration simultaneously using a phase coexistence model of $P 4 \mathrm{~mm}$ and $R 3 \mathrm{~m}$. A good agreement has been reached between the calculated strain and the macroscopically observed one. The deconvoluted features of the individual phase indicate the significantly different contributions of $P 4 \mathrm{~mm}$ and $R 3 \mathrm{~m}$ phases. The tetragonal phase contributes most of the domain switching strain, and the predominant lattice strain is from the rhombohedral phase. The field-induced reversible strain mechanisms of PT-36BS are dominated by intrinsic lattice other than extrinsic domain contribution. Although the PT-36BS is dominated by a tetragonal $P 4 \mathrm{~mm}$ phase, the rhombohedral phase plays a significant role in both reversible field-induced strain and irreversible remanent strain by virtue of the electric field-induced phase transformation. A high remanent strain was observed in PT-36BS ceramics, and it was proved to be mainly controlled by the extrinsic domain switching. Possible methods to release the potential strain are proposed to be appropriate element doping, controlling the relative phase fraction through precisely modifying the composition or improving the process, and con- structing a core-shell structure to provide restoring force.

Received 7 April 2021; accepted 10 June 2021;

published online 20 August 2021

1 Jaffe B, Cook Jr. WR, Jaffe H. Piezoelectric Ceramics. London: Academic Press, 1971

$2 \mathrm{Wu}$ J, Gao X, Chen J, et al. Review of high temperature piezoelectric materials, devices, and applications. Acta Phys Sin, 2018, 67: 207701

3 Hou Y, Zhao H, Zheng M, et al. BSPT-based high temperature piezoelectric materials: Opportunities and challenges. J B Univ Technol, 2020, 46: 664-679

4 Eitel RE, Randall CA, Shrout TR, et al. New high temperature morphotropic phase boundary piezoelectrics based on $\mathrm{Bi}(\mathrm{Me}) \mathrm{O}_{3}-\mathrm{PbTiO}_{3}$ ceramics. Jpn J Appl Phys, 2001, 40: 5999-6002

5 Zhao $\mathrm{H}$, Hou Y, Yu X, et al. A wide temperature insensitive piezoceramics for high-temperature energy harvesting. J Am Ceram Soc, 2019, 102: 5316-5327

$6 \mathrm{Fu} \mathrm{H}$, Cohen RE. Polarization rotation mechanism for ultrahigh electromechanical response in single-crystal piezoelectrics. Nature, 2000, 403: 281-283

7 Schmitt LA, Schönau KA, Theissmann R, et al. Composition dependence of the domain configuration and size in $\mathrm{Pb}\left(\mathrm{Zr}_{1-x} \mathrm{Ti}_{x}\right) \mathrm{O}_{3}$ ceramics. J Appl Phys, 2007, 101: 074107

8 Liu $\mathrm{H}$, Chen J, Huang $\mathrm{H}$, et al. Role of reversible phase transformation for strong piezoelectric performance at the morphotropic phase boundary. Phys Rev Lett, 2018, 120: 055501

9 Liu H, Huang H, Fan L, et al. Synergy between phase transformation and domain switching in two morphotropic phase boundary ferroelectrics. Phys Rev Mater, 2018, 2: 111403

10 Khatua DK, Lalitha KV, Fancher CM, et al. Anomalous reduction in domain wall displacement at the morphotropic phase boundary of the piezoelectric alloy system $\mathrm{PbTiO}_{3}-\mathrm{BiScO}_{3}$. Phys Rev B, 2016, 93: 104103

11 Lalitha KV, Fitch AN, Ranjan R. Correlation between enhanced lattice polarizability and high piezoelectric response in $\mathrm{BiScO}_{3}-\mathrm{PbTiO}_{3}$. Phys Rev B, 2013, 87: 064106

12 Eitel RE, Zhang SJ, Shrout TR, et al. Phase diagram of the perovskite system (1-x) $\mathrm{BiScO}_{3}-x \mathrm{PbTiO}_{3}$. J Appl Phys, 2004, 96: 2828-2831

13 Datta K, Walker D, Thomas PA. Structural investigations of the bismuth scandate-lead titanate $x \mathrm{BiScO}_{3}-(1-x) \mathrm{PbTiO}_{3}$ solid solution for: $0.10 \leq x \leq 0.40$. Phys Rev B, 2010, 82: 144108

14 Kim B, Tong P, Kwon D, et al. Temperature-dependent neutron diffraction study of phase separation at morphotropic phase boundary in $(1-x) \mathrm{BiScO}_{3}-x \mathrm{PbTiO}_{3}$. J Appl Phys, 2009, 105: 114101

15 Hungría T, Houdellier F, Algueró M, et al. Monoclinic symmetry of twin-free nanocrystals in the $\mathrm{BiScO}_{3}-\mathrm{PbTiO}_{3}$ solid solution as revealed by aberration-corrected TEM. Phys Rev B, 2010, 81: 100102

16 Khatua DK, Lalitha KV, Fancher CM, et al. Coupled domain wall motion, lattice strain and phase transformation in morphotropic phase boundary composition of $\mathrm{PbTiO}_{3}-\mathrm{BiScO}_{3}$ piezoelectric ceramic. J Appl Phys, 2016, 120: 154104

17 Pramanick A, Damjanovic D, Daniels JE, et al. Origins of electro-mechanical coupling in polycrystalline ferroelectrics during subcoercive electrical loading. J Am Ceramic Soc, 2011, 94: 293-309

18 Hinterstein M, Lee KY, Esslinger S, et al. Determining fundamental properties from diffraction: Electric field induced strain and piezoelectric coefficient. Phys Rev B, 2019, 99: 174107

19 Hinterstein M, Hoelzel M, Rouquette J, et al. Interplay of strain mechanisms in morphotropic piezoceramics. Acta Mater, 2015, 94: 319327

20 Khansur NH, Hinterstein M, Wang Z, et al. Electric-field-induced strain contributions in morphotropic phase boundary composition of $\left(\mathrm{Bi}_{1 / 2} \mathrm{Na}_{1 / 2}\right) \mathrm{TiO}_{3}-\mathrm{BaTiO}_{3}$ during poling. Appl Phys Lett, 2015, 107: 242902

21 Hall DA, Steuwer A, Cherdhirunkorn B, et al. A high energy synchrotron X-ray study of crystallographic texture and lattice strain in soft lead zirconate titanate ceramics. J Appl Phys, 2004, 96: 4245-4252 
22 Kungl H, Theissmann R, Knapp M, et al. Estimation of strain from piezoelectric effect and domain switching in morphotropic PZT by combined analysis of macroscopic strain measurements and synchrotron X-ray data. Acta Mater, 2007, 55: 1849-1861

23 Liu H, Sun S, Pan Z, et al. Multiple contributions to electrostrain in high performance $\mathrm{PbTiO}_{3}-\mathrm{Bi}\left(\mathrm{Ni}_{1 / 2} \mathrm{Hf}_{1 / 2}\right) \mathrm{O}_{3}$ piezoceramics triggered by phase transformation. J Eur Ceramic Soc, 2019, 39: 5277-5284

24 Hinterstein M, Knapp M, Hölzel M, et al. Field-induced phase transition in $\mathrm{Bi}_{1 / 2} \mathrm{Na}_{1 / 2} \mathrm{TiO}_{3}$-based lead-free piezoelectric ceramics. J Appl Crystlogr, 2010, 43: 1314-1321

25 Fan L, Chen J, Ren Y, et al. Unique piezoelectric properties of the monoclinic phase in $\mathrm{Pb}(\mathrm{Zr}, \mathrm{Ti}) \mathrm{O}_{3}$ ceramics: Large lattice strain and negligible domain switching. Phys Rev Lett, 2016, 116: 027601

26 Lee KY, Shi X, Kumar N, et al. Electric-field-induced phase transformation and frequency-dependent behavior of bismuth sodium titanatebarium titanate. Materials, 2020, 13: 1054

27 Jones JL, Hoffman M, Bowman KJ. Saturated domain switching textures and strains in ferroelastic ceramics. J Appl Phys, 2005, 98: 024115

28 Pramanick A, Daniels JE, Jones JL. Subcoercive cyclic electrical loading of lead zirconate titanate ceramics II: Time-resolved X-ray diffraction. J Am Ceramic Soc, 2009, 92: 2300-2310

29 Jones JL, Slamovich EB, Bowman KJ. Domain texture distributions in tetragonal lead zirconate titanate by X-ray and neutron diffraction. J Appl Phys, 2005, 97: 034113

30 Fan L, Zhang L, Ren Y, et al. Relationship among the crystal structure, texture, and macroscopic properties of tetragonal $(\mathrm{Pb}, \mathrm{La})(\mathrm{Zr}, \mathrm{Ti}) \mathrm{O}_{3}$ ferroelectrics investigated by in situ high-energy synchrotron diffraction. Inorg Chem, 2020, 59: 13632-13638

31 Li JY, Rogan RC, Ustündag E, et al. Domain switching in polycrystalline ferroelectric ceramics. Nat Mater, 2005, 4: 776-781

32 Jones JL, Iverson BJ, Bowman KJ. Texture and anisotropy of polycrystalline piezoelectrics. J Am Ceramic Soc, 2007, 90: 2297-2314

33 Fan L, Chen J, Ren Y, et al. Structural correlation to piezoelectric and ferroelectric mechanisms in rhombohedral $\mathrm{Pb}(\mathrm{Zr}, \mathrm{Ti}) \mathrm{O}_{3}$ ceramics by in situ synchrotron diffraction. Inorg Chem, 2018, 57: 3002-3007

34 Hinterstein M, Rouquette J, Haines J, et al. Structural description of the macroscopic piezo- and ferroelectric properties of lead zirconate titanate. Phys Rev Lett, 2011, 107: 077602

35 Choi SY, Jeong SJ, Lee DS, et al. Gigantic electrostrain in duplex structured alkaline niobates. Chem Mater, 2012, 24: 3363-3369

Acknowledgements This work was supported by the National Natural Science Foundation of China (21825102, 22075014 and 12004032), the Fundamental Research Funds for the Central Universities, China (06500162), the National Postdoctoral Program for Innovative Talents (BX20200044), and German Research Society (DFG) (HI 1867/1-2). The use of the Advanced Photon Source at Argonne National Laboratory was supported by the U. S. Department of Energy, Office of Science, Office of Basic Energy Sciences, under Contract No. DE-AC02-06CH11357. We appreciate the selfless help and encouragement from Yaping Cao.

Author contributions Sun S prepared the samples with support from Liu $\mathrm{H}$ and carried out the dielectric, ferroelectric, and SEM measurements. Liu H performed the in situ SXRD experiments with the assistance of Ren Y. Hinterstein $M$ analyzed the in situ data using the STRAP method. Sun S wrote the paper with support from Liu H, Hinterstein M and Chen J. All authors contributed to the general discussion.

Conflict of interest The authors declare that they have no conflict of interest.

Supplementary information Detailed calculation process for the overall remanent strain $\left(S^{\mathrm{Rem}}\right)$ and the reversible strain $\left(S^{\mathrm{Rev}}\right)$, and supporting data are available in the online version of the paper.
Shengdong Sun obtained his BS degree from the University of Science and Technology Beijing (USTB). He is currently a $\mathrm{PhD}$ candidate at USTB. His research interests include ferroelectric and piezoelectric materials, piezoelectric mechanisms, and high-temperature piezoceramics.

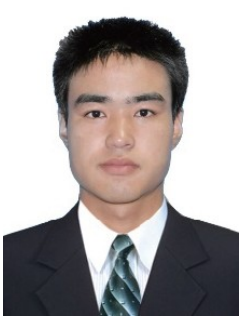

Hui Liu received his PhD degree from USTB in 2020 From 2018 to 2019, he worked as a visiting scholar at the University of Central Michigan and Argonne National Laboratory in the United States. He joined USTB as a distinguished associate professor in 2020 His research interest is the performance and mechanism of ferroelectric oxides.

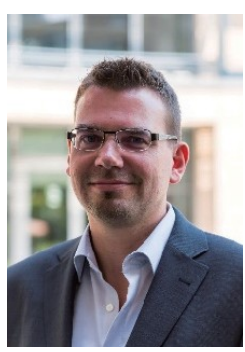

Manuel Hinterstein holds the Emmy Noether-Group leader at Karlsruhe Institute of Technology Institute of Applied Materials, Karlsruhe Institute of Technology, and he is also an adjunct senior lecturer at the School of Materials Science and Engineering, University of New South Wales (Sydney, AU). He received his $\mathrm{PhD}$ degree from Technische Universität Darmstadt Materials Science in 2010 and he researched at Technische Universität Dresden Institut für Werkstoffwissenschaft as postdoc from 2010 to 2013. He is interested in functional electroceramics, with focus on the 'positive temperature coefficient of resistance' (PTCR) effect, as well as sensor, actuator and electrocaloric applications.

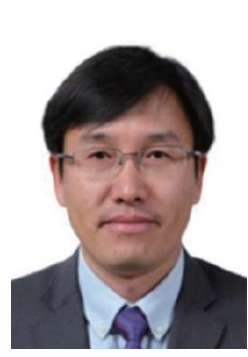

Jun Chen is currently a full professor at USTB, China $\mathrm{He}$ received his BSc in materials science and technology from Hefei University of Technology, China, in 2001, and obtained his $\mathrm{PhD}$ in metallurgical physical chemistry from USTB, China, in 2007. In 20082009, he was financially supported by the Alexander von Humboldt Fellowship to research in the field of electrical ceramics at TU-Darmstadt, Germany. He visited the Structures Laboratory of the Tokyo Institute of Technology, Japan, as the foreign guest professor in 2015, and visited the Department of Physics and Astronomy of University of Padova, Italy as a visiting scientist in 2019. His current research interests include crystal structure, local structure, structure-property relationships, and new materials design for ferroelectric, piezoelectric, magnetic, and negative thermal expansion solids.

\section{揭示相共存体系 $\mathrm{PbTiO}_{3}-\mathrm{BiScO}_{3}$ 中内在和外在压电贡献}

孙胜东 ${ }^{1,2}$, 刘 辉 $3^{*}$, 任洋 ${ }^{5}$, Manuel Hinterstein $4^{*}$, 陈骏 $1,2,3^{*}$

摘要 $\mathrm{PbTiO}_{3}-\mathrm{BiScO}_{3}$ 陶瓷兼具高压电系数和高居里温度, 在高温传感 器和制动器领域极具应用潜力. 探明潜在的压电机理对其应用具有重 要作用. 本文结合先进原位电场高能同步辐射技术和STRAP分析方法, 定量揭示了 $\mathrm{PbTiO}_{3}-\mathrm{BiScO}_{3}$ 体系相界附近较高应变的内在和外在贡献. 观察到在共存的四方相和三方相之间发生了电场诱导的相转变. 实验 发现畴反转应变主要来源于四方相, 而占主导作用的晶格应变主要来 源于少量共存的三方相. 电场诱导了相含量的变化, 定量分析结果表明 该体系中可逆应变主要源于内在的晶格应变. 相反, 不可逆的畴反转应 变导致了较大的剩余应变. 现有结论表明, 将剩余应变转化为可逆应变 将是提高压电性能的战略方向. 本研究有助于进一步理解高压电性能 并有望推进 $\mathrm{PbTiO}_{3}-\mathrm{BiScO}_{3}$ 体系的应用. 